\title{
Avaliação dos Cursos de Extensão Universitária realizados pela Facul- dade de Medicina de Ribeirão Preto da USP entre 2002 e 2008
}

\section{Evaluation of the University Extension Courses promoted by the Medi- cal School of Ribeirão Preto of the University of São Paulo between 2002 and 2008}

Antonio Pazin Filho ${ }^{1}$, Anette Hoffmann², Eucleia Primo Betioli Contel ${ }^{3}$, Maria de Lourdes Veronese Rodrigues ${ }^{4}$

\begin{abstract}
RESUMO:
Introdução: A RESOLUÇÃO da Comissão de Cultura e Extensão da Universidade de Sào Paulo- CoCEx5072 da Universidade de São Paulo(USP) regulamentou os cursos de extensão universitária. A Faculdade de Medicina de Ribeirão Preto da USP(FMRP-USP) passou a incentivar que diversos cursos nãoregulamentados fossem oficializados. Diversos empecilhos foram detectados, sendo necessário reavaliar o processo.

Metodologia: Foram avaliados 106 cursos de extensão universitária realizados entre 2002 e 2008. Obteve-se dados referentes ao tipo de curso, Departamento responsável, regularidade e número de vagas oferecidas, comparando-se com a situação dos cursos no Sistema Apolo-USP. O trâmite dos processos foi avaliado através da diferença em dias entre o início e o final do processo. Todos os cursos que tiveram os certificados emitidos e anexados ao processo foram classificados como concluídos.

Resultados: 1) O número de cursos regulamentados realizados pela FMRP-USP é baixo, sendo muito inferior à sua potencialidade; 2) A maioria dos cursos compreende cursos de curta duração (Difusão) e são reproduzidos em caráter anual; 3) A Comissão de Cultura e Extensão da FMRP-USP não dispõe de pessoal suficiente para gerenciamento dos cursos, dificultando ações de orientação e tramitação de processos.

Conclusões: A infra-estrutura para o gerenciamento dos cursos de extensão da FMRP-USP precisa ser melhorada. Nitidamente, a demanda atual, que é pequena, não está sendo atendida na totalidade exigida com prejuízos sobre a qualidade. Esta situação pode comprometer de modo importante a pretensão de se ampliar o cadastro de novos cursos de extensão. Medidas urgentes de estruturação para o gerenciamento adequado destes cursos devem ser adotadas e os cursos anteriores já cadastrados e não concluídos devem ser reavaliados e finalizados.
\end{abstract}

Palavras-chave: Educação de Pós-Graduação. Cursos/Difusão. Apoio ao Desenvolvimento de Recursos Humanos. Educação Continuada. Regulamentação USP. Instituições de Ensino. Documentação.

1 Docente da Divisão de Emergências Clínicas do Departamento de Clínica Médica da Faculdade de Medicina de Ribeirão Preto da Universidade de São Paulo e membro da Comissão de Cultura e Extensão da FMRP-USP.

2 Docente do Departamento de Fisiologia e membro da Comissão de Cultura e Extensão da FMRP-USP.

3 Docente do Departamento de Genética e Vice-Presidente da Comissão de Cultura e Extensão da FMRP-USP.

4 Docente do Departamento de Oftalmologia, Otorrinolaringologia e Cirurgia de Cabeça e Pescoço e Presidente da Comissão de Cultura e Extensão da FMRP-USP.
Correspondência: Prof. Dr. Antonio Pazin-Filho R. Bernardino de Campus, 1000 14010-030 - Ribeirão Preto/SP Telefone - 16-3610-1110 apazin@fmrp.usp.br

Artigo recebido em 1\%/04/2010 Aprovado 20/09/2010 
Mediante a RESOLUÇÃO DA COMISSÃO DE CULTURA E EXTENSÃ̃ - CoCEX No ${ }^{\circ}$ 5072, DE 16 DE SETEMBRO DE 2003, a Universidade de São Paulo regulamentou a estruturação de cursos de extensão universitária. ${ }^{1}$ Em consonância com esta resolução, a Faculdade de Medicina de Ribeirão Preto da Universidade de São Paulo (FMRP-USP) passou a incentivar que diversos cursos não-regulares, promovidos fora do contexto de graduação ou pósgraduação fossem oficializados. ${ }^{2,3}$

Recentemente, a inauguração do Espaço de Cultura e Extensão Universitária da FMRP-USP (ECEU-FMRP-USP) concretizou o esforço da Comissão de Cultura e Extensão em fomentar eventos que aproximem a USP da comunidade. Cabe agora o desafio da implementação de diversas medidas necessárias para manter e progredir na correta utilização deste espaço. Em reunião ordinária da Comissão de Cultura e Extensão da FMRP-USP em que a utilização deste espaço estava sendo discutida, levantouse a possibilidade de encorajar o seu uso pelos docentes interessados em ministrar cursos de extensão.Neste processo, é natural o incentivo para que estes cursos sejam realizados na forma de cursos de extensão universitária, seguindo as diretrizes da resolução CoCex $\mathrm{N}^{\circ}$ 5072. Na discussão inicial de um regulamento para utilização dos espaços do ECEU que se inicia na Comissão de Cultura e Extensão, já se discutiu o critério de priorizar a reserva de salas para os eventos que sejam cadastrados como cursos de extensão.

Paralelamente a este processo, tem sido discutida a possibilidade de se formalizar junto à USP as atividades docentes de áreas clínicas em programas de residência médica através deste processo. Tem sido empenho da Comissão de Cultura e Extensão da FMRPUSP avaliar estas possibilidades, muito embora a Resolução CoCex No 5072 possa limitar esta solução.

Em que pese todos estes fatores, diversos empecilhos foram detectados ao longo destes quase sete anos de cadastro dos cursos de extensão, sendo necessária uma reavaliação do processo. Este trabalho tem como objetivo avaliar os 106 cursos cadastrados entre 2002 e 2008 para propiciar uma análise crítica e atitudes corretivas do processo, de modo a permitir à Comissão de Cultura e Extensão decisões instruídas sobre este assunto.

\section{Metodologia}

Foram levantados os 106 cursos de extensão universitária realizados entre 2002 e 2008 cadastra- dos junto à Comissão de Cultura e Extensão da FMRPUSP. Para estes cursos obteve-se dados referentes ao tipo de curso, Departamento responsável, regularidade dos cursos e número de vagas oferecidas. Paralelamente, obteve-se a situação dos cursos oferecidos e cadastrados no Sistema Apolo de Cultura e Extensão Universitária da Universidade de São Paulo. ${ }^{4}$

Os cursos foram classificados em relação à regularidade na dependência de existir mais de uma versão do curso, ou seja, se ele foi oferecido mais do que uma vez ao longo do período de estudo.

Para os anos de 2002 a 2007, os dados sobre o trâmite dos processos foram mantidos em banco de dados desenvolvido em plataforma Microsoft Access. Neste banco, cada etapa da tramitação do processo era anexada ao número do processo, documentandose a data da entrada e o procedimento executado. A partir do ano de 2008, este procecimento foi abandonado e os cursos registrados em planilha de Microsof Excel.

Para analisar o trâmite dos processos, calculou-se a diferença em dias entre a primeira entrada (início do processo) e a última entrada (final do processo) no banco de dados para cada curso. Todos os cursos que tiveram os certificados emitidos e anexados ao processo foram classificados como concluídos. Foram considerados para esta análise apenas os cursos de Difusão e realizados após 2004 (antes de 2004 existem apenas 3 cursos cadastrados) e até 2007 (em 2008 a documentação foi interrompida).

A análise dos dados e elaboração dos gráficos foi realizada com o programa STATA. ${ }^{5}$

\section{Resultados}

Entre 2002 e 2008, foram realizados 106 cursos de extensão universitária na FMRP-USP, sendo $78(73,6 \%)$ na modalidade Difusão, $8(7,5 \%)$ como Especialização, $9(8,5 \%)$ como Atualização e 11 $(10,4 \%)$ como Aperfeiçoamento - Figura 1. De particular interesse, é que apenas $5(4,7 \%)$ foram oferecidos em caráter de inscrição taxada, gerando recursos financeiros para a Universidade.

A Figura 2A demonstra a distribuição dos cursos de Difusão e dos outros tipos de curso de acordo com o ano do período de estudo. A partir de 2004 houve um incremento no número de cursos oferecidos, sendo que a FMRP-USP ofereceu uma média de 15,7 cursos por ano (variando entre 1 e 25 cursos o número total de cursos em cada ano). Também pode ser observado na Figura 2A que os outros tipos de curso 


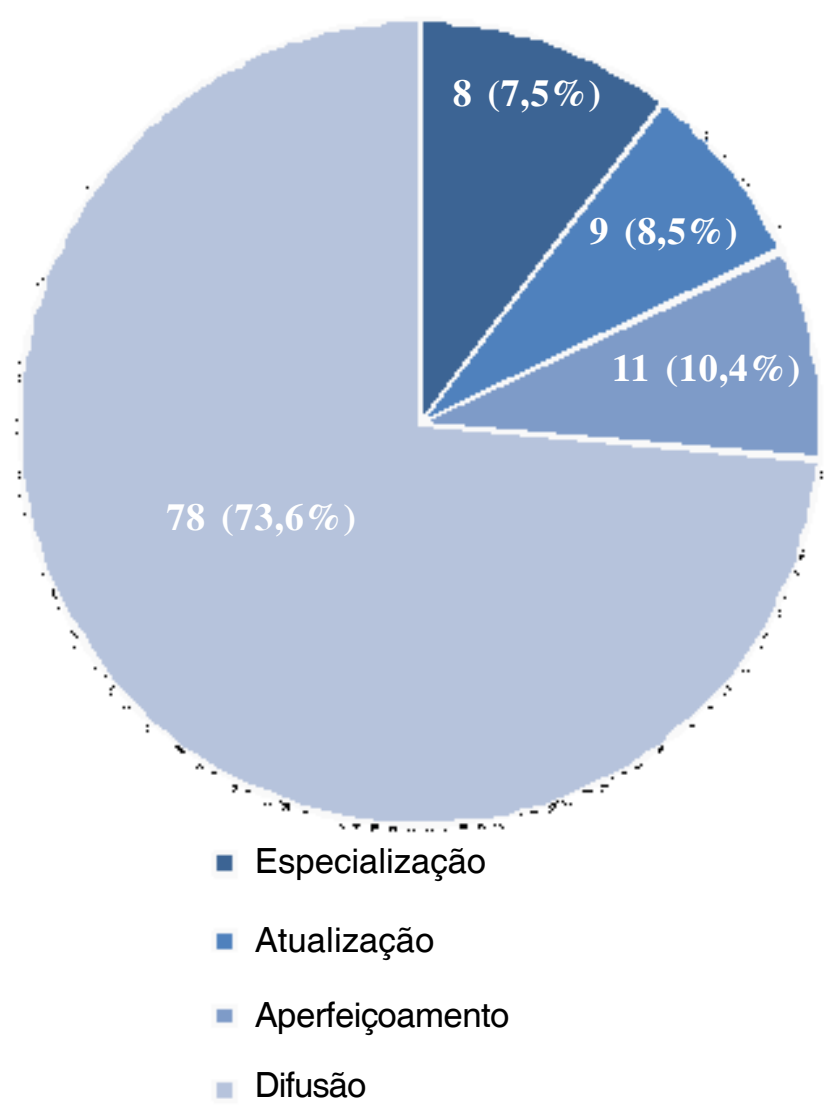

Figura 1: Distribuição dos cursos de extensão realizados na FMRPUSP entre 2002 e 2008 de acordo com a modalidade.

(Especialização, Atualização e Aperfeiçoamento) foram expressivamente em menor número que os de Difusão e que eles vêm diminuindo ao longo dos anos. Em 2008, apenas 3 dos 15 cursos oferecidos não foram de Difusão.

A Figura 2B demonstra a distribuição dos cursos de acordo com a regularidade de oferecimento, entendida para efeitos de análise como a repetição do curso em mais de um ano ao longo do período de estudo. Pode-se observar que cerca de 12 a 15 cursos são regulares por ano e que novas versões de curso não ultrapassam 8 cursos, tendo decaído para 5 em 2008.

A Figura 3 ilustra a distribuição do número de cursos de acordo com o Departamento responsável pela sua realização.

Todos os cursos levantados junto à Comissão de Cultura e Extensão estão efetivamente cadastrados no Sistema Apolo. Quando se procede para a análise dos dados cadastrados junto à Comissão de Cultura e Extensão entre 2004 e 2007, observa-se, no entanto, que dos 85 cursos considerados, apenas 51
(60\%) tiveram certificação expedida - Figura 4. Além disso, a certificação diminuiu drasticamente no ano de 2007, sendo que apenas 5 (20\%) dos 20 cursos realizados foram efetivamente concluídos.
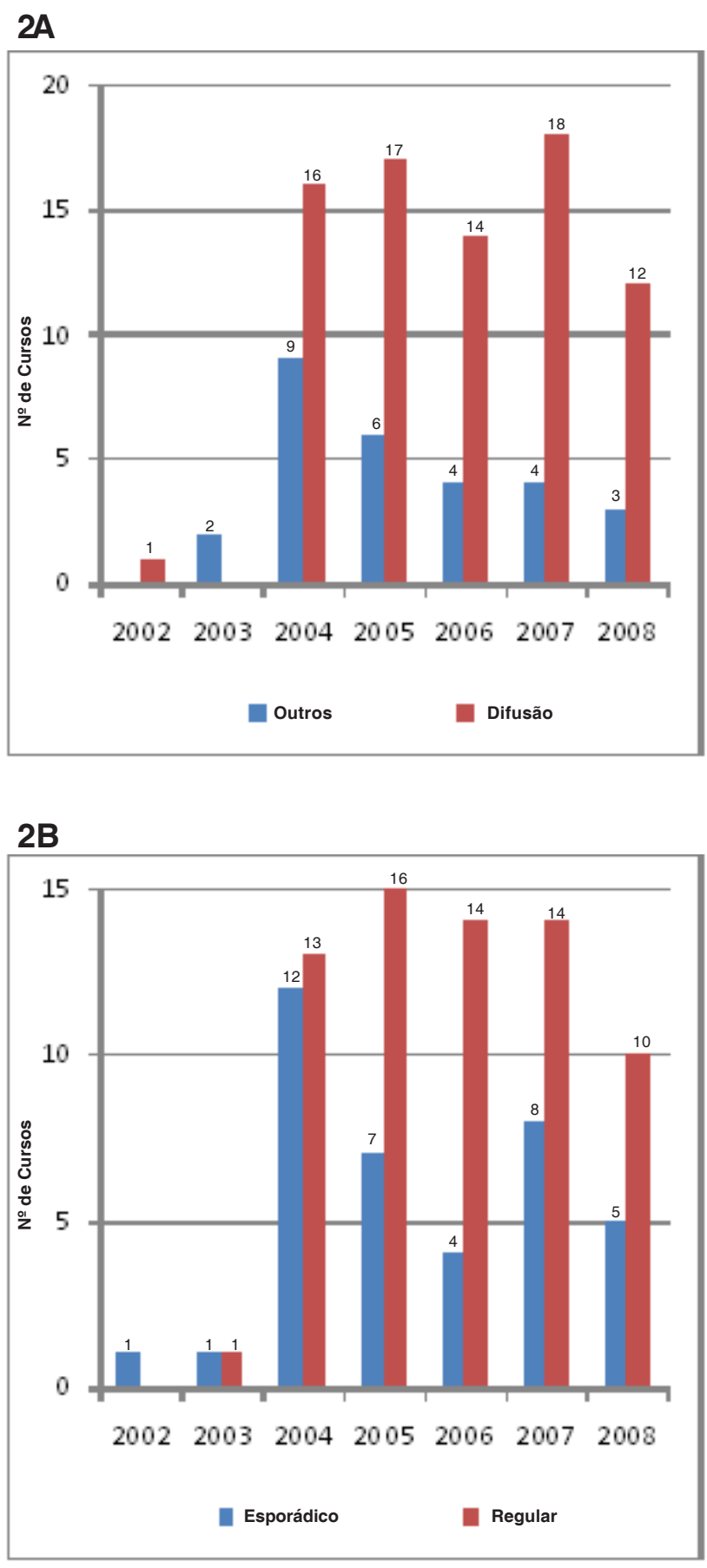

Figura 2: Distruição do número de cursos de extensão por ano de realização de acordo com a modalide (A) - Difusão ou Outros (Especialização, Atualização ou Aperfeiçoamento) - e com a periodiciadade (B) - Esporádico (realizado apenas uma vez) ou Regular (realizado mais de uma vez). 


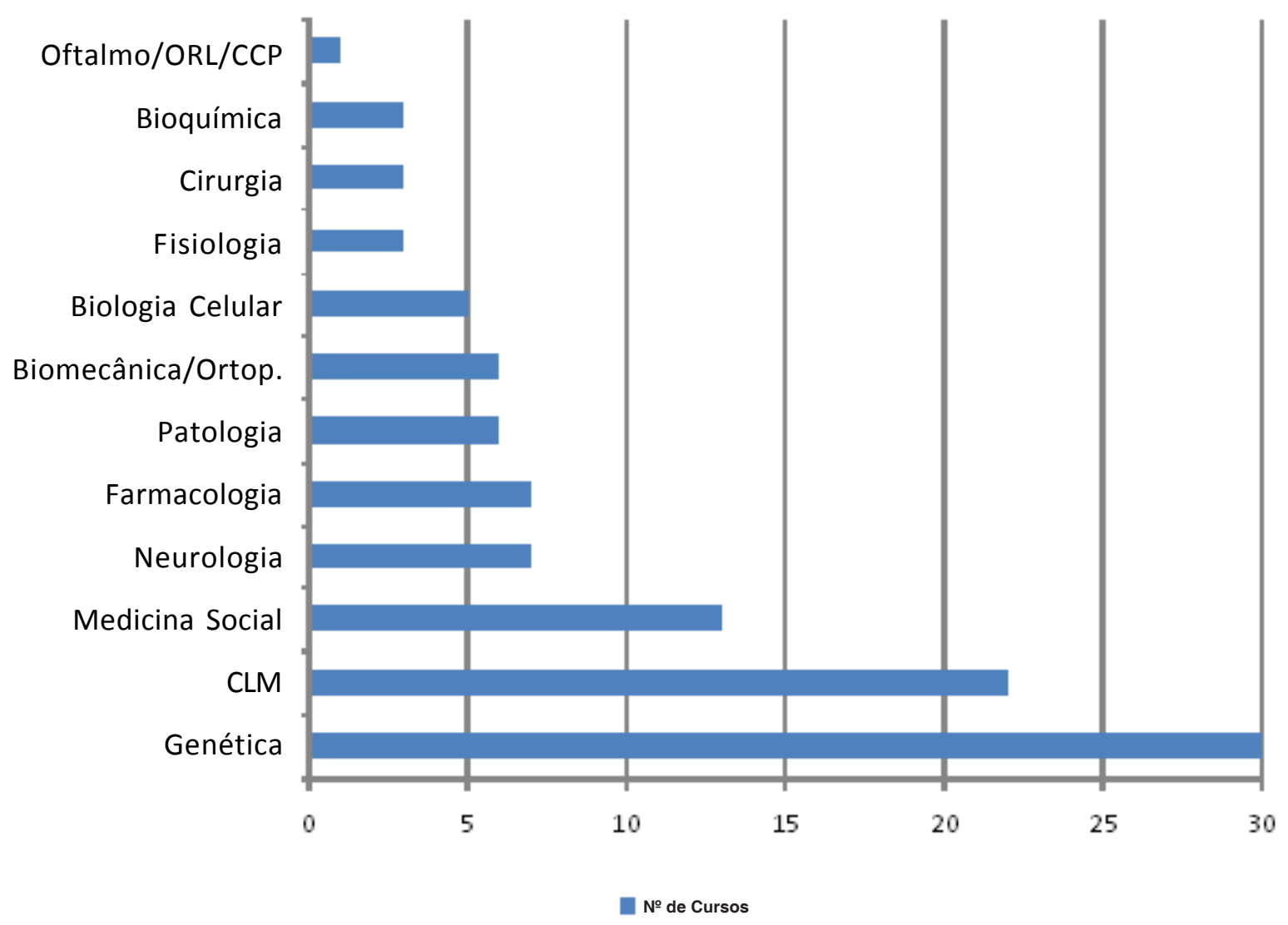

Figura 3: Distribuição do número de cursos de extensão realizados entre 2002 e 2008 de acordo com o Departamento.

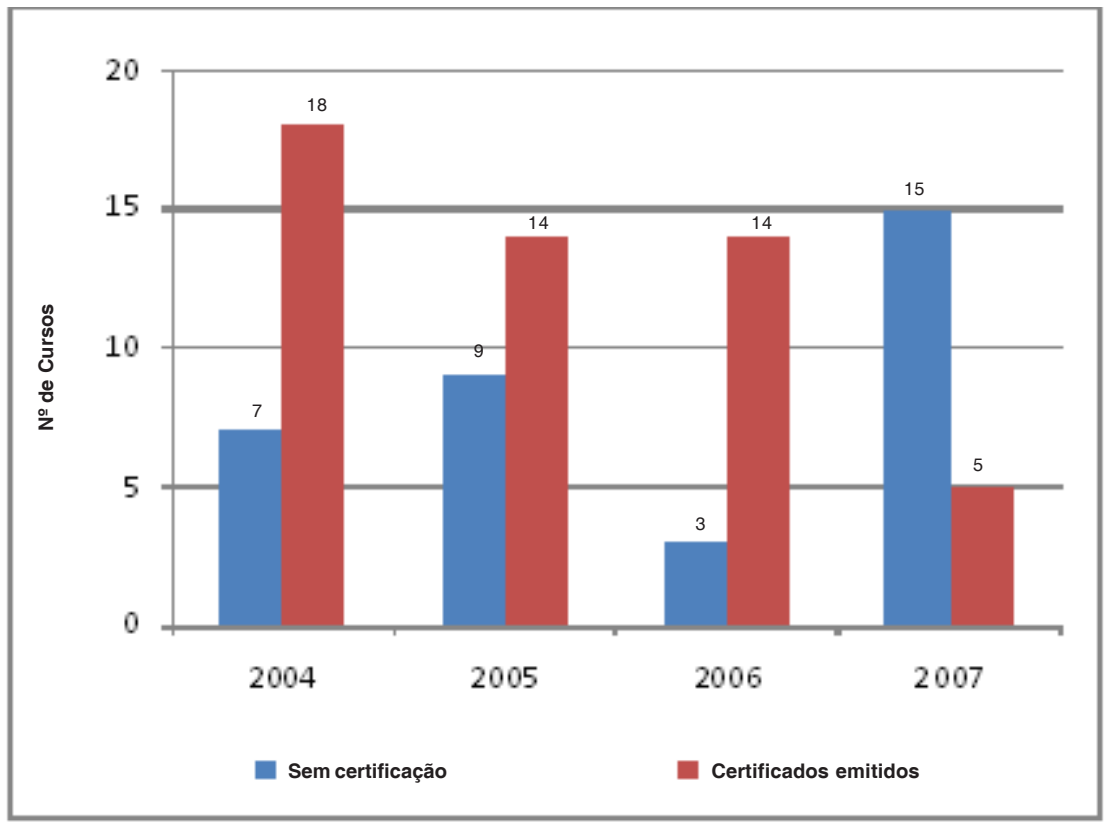

Figura 4: Distribuição do número de cursos de extensão de acordo com a expedição do certificado entre os anos de 2004 e 2007. 
O tempo decorrido para tramitação dos processos para os cursos de Difusão entre 2004 e 2007 está ilustrado na Figura 5. Pode-se observar que ao longo dos quatro anos de análise, o tempo decorrido para tramitação do processo diminui de modo importante, tanto para os cursos regulares como para os cursos novos.

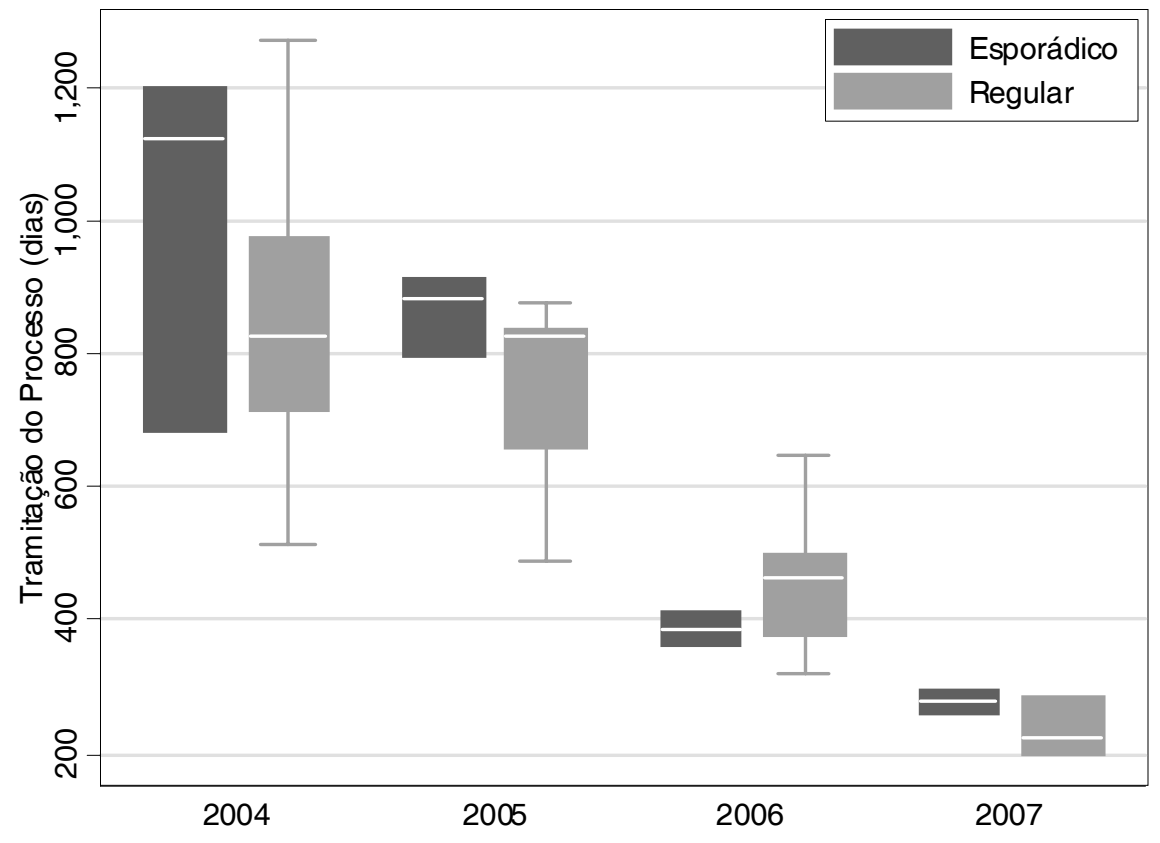

Figura 5: Box-plot para a duração da tramitação do processo para os cursos de Difusão realizados entre 2004 e 2007 que foram concluídos (tiveram certificado emitido).

tes burocráticos, envolvendo a Pró-Reitoria de Cultura e Extensão. Deve ser ressaltado que, além do baixo número de cursos de outras modalidades realizados, estes estão decaindo numericamente de ano a ano.

Também chama a atenção que apenas 5 dos 106 cursos realizados exigiam taxa de inscrição dos alunos envolvidos, gerando recursos extraorçamentários para a USP e para a Comissão de Cultura e Extensão da FMRP-USP. Cursos pagos geralmente requerem mais envolvimento, exigindo maior estrutura de secretaria para seu gerenciamento e elaboração do orçamento detalhado com prestação de contas. Tais facilidades não estão disponíveis atualmente junto à Comissão de Cultura e Extensão, a quem compete a análise do mérito e da documentação dos cursos.

Um ponto preocupante é que embora a FMRPUSP esteja mantendo o número de cursos oferecidos por ano, estes são decorrentes do esforço de poucos docentes

\section{Discussão}

O presente levantamento demonstra que apenas uma média de 15,7 cursos por ano são cadastrados como cursos de extensão pela FMRP-USP, apesar do elevado número de atividades desenvolvidas pelos seus docentes fora do contexto da graduação e pós-graduação, principalmente pelo envolvimento com a residência médica. Infelizmente, não é possível obter dados objetivos do quanto representam estas atividades desenvolvidas que poderiam ser documentadas na forma de cursos de extensão, muito embora haja algumas documentações isoladas. ${ }^{6}$ Provavelmente, este foi um dos intuitos da USP ao promulgar a Resolução CoCex No 5072.

Um outro ponto que merece destaque é que a maioria dos cursos realizados são cursos de curta duração, sendo portanto cadastrados como cursos de Difusão. As modalidades de Especialização, Aperfeiçoamento e Atualização são de maior duração e requerem maior envolvimento com a elaboração e ministração do curso. Também há necessidade de mais trâmi- que oferecem o mesmo curso com regularidade anual. Os motivos para este baixo envolvimento na realização de cursos credenciados provavelmente é multifatorial e, certamente, não podem ser totalmente esclarecidos a partir dos dados do presente levantamento. No entanto, algumas hipóteses podem ser levantadas e que deveriam merecer a atenção e intervenção da Comissão de Cultura e Extensão e da USP.

O modelo preconizado pela USP na Resolução CoCex No 5072 faz com que os cursos sejam chancelados pela Universidade, mas não beneficiam o promotor, por não ser atividade valorizada como produção docente. Isto com certeza se reflete no empenho dos docentes em cadastrar os cursos. Por vezes, a chancela do HCFMRP-USP é o suficiente para a população-alvo. Iniciativas para se contornar este problema de crédito acadêmico já estão sendo desenvolvidas, através da informatização das atividades no Sistema Artemis, em fase de elaboração.

A Comissão de Cultura e Extensão da FMRPUSP esteve desfalcada de secretária nos últimos dois anos com a aposentadoria da servidora, cuja reposi- 
ção encontra-se em tramitação. Esta situação provavelmente ocasinou dificuldades para auxiliar docentes interessados em cadastrar suas atividades como curso de extensão universitária. Isto também foi muito provavelmente responsável pelo aumento de cursos que não tiveram sua certificação efetivada em 2007.

A burocracia, apesar de problemática nas primeiras ocasiões, uma vez vencida passa a não ser empecilho para a repetição dos cursos, haja vista a persistência de cursos que se repetem ano a ano.

A reconstituição do secretariado da Comissão de Cultura e Extensão da FMRP-USP deve ser encarada como urgente. Uma vez reposta a secretária, medidas para facilitar o processo de cadastramento devem ser implementadas. O treinamento de funcionários dos diversos Departamentos da FMRP-USP para cadastramento dos cursos diretamente no Sistema Apolo facilitaria a tramitação do processo e diminuiria a burocracia. Estas medidas já foram recentemente implementadas, resultando na facilitação do trâmite do processo do curso no sistema e na disponibilização de certificados aos alunos de modo mais ágil.

Recentemente, a ampliação das características dos cursos de extensão, incluindo uma nova modalidade - Prática Profissionalizante e Programa de Atualização, foi uma solução encontrada em outras unidades da USP e já se encontra em implementação na FMRP-USP. Essas normas foram discutidas e aprovadas na $79^{\mathrm{a}}$ Reunião Ordinária da CoCEx-FMRPUSP e referendadas pela CoCEx-USP. A aprovação dessas normas irá simplificar o trâmite de cursos regulares, que poderão ser cadastrados para serem realizados regularmente por um período pré-definido, que pode variar até cerca de dois anos. Isto abre a possibilidade para que os programas de residência médica oferecidos pelo Hospital das Clínicas da FMRP-USP possam ser cadastrados como cursos de extensão, sendo a carga horária dispendida com estes programas contabilizada para os docentes responsáveis.

Finalmente, o desenvolvimento de uma estrutura dentro do ECEU voltada para o gerenciamento destes cursos deve ser perseguida e efetivada. Muitos destes cursos requerem o cadastro atualizado dos ministrantes, com as respectivas aprovações na CERT, o que já está disponível nas diversas esferas da FMRPUSP. O conhecimento mais aprofundado do Sistema Apolo muito provavelmente permitiria efetivar o resgate destas informações de modo mais ágil, diminuindo a burocracia necessária.

Em que pese todas estas considerações, o fortalecimento dos cursos de extensão universitária trará também benefícios e desafios maiores sobre o papel da Universidade. Os cursos de extensão se constituem na possibilidade da Universidade assumir um papel negligenciado de Educação Continuada para profissionais formados, mantendo o elo com egressos e estabelecendo novos elos com outros profissionais da região de inserção. ${ }^{7,8}$ A Educação Continuada está sendo muito debatida atualmente, por ser nítida a sua necessidade para manter o profissional apto a realizar suas funções e também porque os métodos tradicionais empregados (aulas teóricas, cursos de curta duração, palestras, etc) têm sido questionados como pouco eficazes. ${ }^{7,9}$ É necessário que os programas de Educação Continuada respeitem as características de ensino do adulto, utilizando técnicas mais avançadas. ${ }^{10,11,12} \mathrm{O}$ envolvimento com estas técnicas terão impacto para a qualidade dos cursos de graduação e pós-graduação sensu lato oferecidos. ${ }^{6}$

A função da Educação Continuada tem sido realizada principalmente por associações profissionais de diversas naturezas, que sofrem pressões intensas sobre o conteúdo a ser oferecido aos alunos, levando países mais organizados a desenvolverem entidades oficiais de acreditação para garantir a qualidade do que é oferecido. ${ }^{8} \mathrm{O}$ maior envolvimento da Universidade com esta atividade, desde que adequadamente reconhecido, permitirá que a ela assuma a liderança natural que já exerce na graduação e pós-graduação. Também irá fornecer retro-alimentação sobre as demandas profissionais atuais, para que possa adequar melhor seus cursos de graduação e pós-graduação senso latu.

Em resumo, a infraestrutura para o gerenciamento dos cursos de extensão na FMRP-USP precisa ser melhorada. Nitidamente, a demanda atual, que é pequena, não está sendo atendida na totalidade exigida com prejuízos sobre a qualidade. Esta situação pode comprometer de modo importante a pretensão de se ampliar o cadastro de novos cursos de extensão, principalmente a Residência Médica, frente ao volume de trabalho que isto irá impor à Comissão de Cultura e Extensão. Medidas urgentes de estruturação para o gerenciamento adequado destes cursos devem ser adotadas e os cursos anteriores já cadastrados e não concluídos devem ser reavaliados e finalizados.

\section{Agradecimentos}

Os autores agradecem a Sra. Maristela Medeiros Santos da Silva pelo auxílio na identificação dos dados para a realização deste trabalho. 


\section{ABSTRACT}

Background: University of São Paulo(USP) CoCEx-5072 resolution regulates university extension courses. The Medical School of Ribeirão Preto USP(MSRP-USP) has adhered and incentivates documentation of all courses conducted. Several problems were identified, being mandatory process reevaluation.

Metodology: 106 courses conducted between 2002 and 2008 were evaluated. Data regarding course type, duration, regularity and number of attendees were recovered and compared with the official USPApolo web system. Duration of process was calculated through the difference between date of beggining and end of process. All courses were considered concluded when certification was emited.

Results: 1)The number of courses conducted by MSRP-USP is low, much inferior to its potential; 2) The majority of courses comprehends short duration courses (Difusion) conducted on a yearly basis; 3 ) There were problems with personal for guiding and documenting the courses in the MSRP-USP.

Conclusion: The extension courses management in the MSRP-USP should be improved. Even with the low number of courses, guidance and documentation is not properly undergone. This could jeopardize the policy of increasing the number of courses documented. Urgent measures should be implemented for adequate management of the actual demand and increasing documentation.

Key-words: Education, Graduate. Courses /Diffusion. Training Support . Education, Continuing. Regulatory University of São Paulo. Schools. Documentation.

\section{Referências}

1. RESOLUÇÃO CoCEx № 5072, Universidade de São Paulo, (2003).

2. Hoffmann A. Cinqüentenário da Faculdade de Medicina de Ribeirão Preto - USP. Medicina(Ribeirão Preto) 35, 229. 2002. Ref Type: Journal (Full)

3. Veronese Rodrigues ML. Inovações no Ensino Médico e Outras Mudanças: Aspectos Históricos e na Faculdade de Medicina de Ribeirão Preto-USP. Medicina(Ribeirão Preto) 35, 231 235. 2002. Ref Type: Journal (Full)

4. Universidade de São Paulo. Sistema Apolo. Universidade de São Paulo 2009;Available from: URL: https://sistemas.usp.br/ apolo/

5. Stata Statistical Software [computer program]. Version 10.1. College Station, TX: StataCorp; 2009.

6. Pazin-Filho A, Schmidt A, Filipini C, Castro RBP, Rosa RM, Rosa MAF, Bueno CDF, Maciel BC. Simulação de pacientes - Cursos de suporte de vida - ACLS, BLS e PALS na FMRP-USP. Medicina (Ribeirão Preto) 2007;40(2):204-12.
7. Davis D, O'Brien MA, Freemantle N, Wolf FM, Mazmanian P, Taylor-Vaisey A. Impact of formal continuing medical education: do conferences, workshops, rounds, and other traditional continuing education activities change physician behavior or health care outcomes? JAMA 1999 September 1;282(9):867-74.

8. Davis N, Davis D, Bloch R. Continuing medical education: AMEE Education Guide No 35. Med Teach 2008;30(7):65266.

9. Meireles VC, Brito BS, Tome EJ, Bueno SC, Góes HLF. Educação continuada em saúde: refletindo sobre sua importância. Arq Apadec 2004;8(Mai):286-8.

10. Galbraith MW. Adult learning methods: a guide for effective instruction. 1 ed. Malabar (FL): Robert E. Krieger Publishing Company; 1990.

11. Pazin-Filho A. Características do aprendizado do adulto. Medicina (Ribeirão Preto) 2007;40(1):7-16.

12. Pazin-Filho A. Simpósio didática - simulação. Medicina (Ribeirão Preto) 2007;40(2):151. 